\title{
Three-Dimensional Free Vibration Analysis of a Viscothermoelastic Hollow Sphere
}

\author{
Jagan Nath Sharma ${ }^{1}$, Dinesh Kumar Sharma ${ }^{2}$, Sukhjit Singh Dhaliwal ${ }^{2}$ \\ ${ }^{1}$ Department of Mathematics, National Institute of Technology, Hamirpur (HP), India \\ ${ }^{2}$ Department of Mathematics, Sant Longowal Institute of Engineering and Technology, Sangrur, India \\ Email: jns@nitham.ac.in,dksharma200513@gmail.com, sukhjit_d@yahoo.com
}

Received November 18, 2011; revised December 20, 2011; accepted December 30, 2011

\begin{abstract}
This paper concentrates on the study of the three-dimensional free vibrations in a homogenous isotropic, viscothermoelastic hollow sphere whose surfaces are subjected to stress free, thermally insulated or isothermal boundary conditions. The use of governing partial differential equations is solved into a coupled system of ordinary differential equations. The equation for toroidal motion gets decoupled from rest of the motion and remains unaffected due to thermal variations. Matrix Fröbenious method of extended power series is employed to obtain the solution. The secular equations for the existence of various types of possible modes of vibrations in the considered hollow sphere are derived in the compact form. The special cases of spheroidal and toroidal modes of vibrations of a hollow sphere have also been deduced and discussed. In order to explore the characteristics of vibrations the secular equations are further solved by using fixed point iteration numerical technique with the help of MATLAB software tools. The computer simulated results have been presented graphically for copper material.
\end{abstract}

Keywords: Toroidal; Spheroidal; Isotropic; Viscothermoelastic Vibrations; Spherical Structures

\section{Introduction}

The exact three-dimensional analysis of free vibrations of elastic spherical structures is well established in [1-4]. The coupled theory of thermoelasticity proposed by Lord and Shulman [5] incorporates a flux-rate term into the Fourier Law of heat conduction and involves a hyperbolic-type heat transport equation admitting wave type thermal signals. Green and Lindsay [6] formulated temperature-rate-dependent thermoelasticity by introducing relaxation time that reckons a finite speed of heat propagation. Hetnarski and Ignaczac [7] studied the response of a semi-space due to a short laser pulse in context of generalized thermoelasticity. Buchanan and Ramirez [8] computed the free vibration frequencies for solid ellipsoids by using Ritz method. Sharma and Sharma [9] studied vibrations of a transradially isotropic coupled thermoelastic solid sphere by using matrix Fröbenius method. Neuringer [10] developed the procedure of Fröbenius method when the roots of indicial equation are complex. Several mathematical models $[11,12]$ have been used to accommodate the energy dissipation is due to internal friction in vibrating viscoelastic solids. Moreover the Kelvin-Voigt model is one of the macroscopic mechanical models which is also used to describe the viscoelastic behavior of a material. Mukhopadhyay [13] studied the effect of thermal relaxation time on viscothermoelastic interactions in an unbounded body with a spherical cavity subjected to periodical loading. Sharma [14] investigated the propagation of waves in an infinite Kelvin-Voigt type viscoelastic plate in the context of coupled thermoelasticity.

This paper is devoted to the exact three-dimensional vibration analysis of homogenous isotropic, viscothermoelastic hollow sphere subjected to 1) stress free thermally insulated and 2) stress free isothermal conditions. The potential function technique has been employed to decouple purely shear motion which remains independent of thermal variations. Upon using separation of variable technique, the problem is reduced to a system of four ordinary differential equations. In order to obtain frequency equation as second class (spheroidal) vibrations the coupled system have been solved by using Matrix FRÖBENIUS series method. The fixed point iteration numerical technique with the help of MATLAB software tools is used to compute frequency and damping of the vibrations. The computer simulated results in respect of lowest frequency, dissipation factor, stresses, displacements and temperature change have been presented graphically of the hollow sphere.

\section{Mathematical Model}

We consider a homogenous isotropic thermally conducting, viscothermoelastic hollow sphere of outer radius $a$ 
and inner radius $b$ initially at uniform temperature $T_{0}$ in the undisturbed state. The basic governing equations of motion and heat conduction for displacement

$\boldsymbol{u}(r, \theta, \phi, t)=\left(u_{r}, u_{\theta}, u_{\phi}\right)$ and temperature change

$T(r, \theta, \phi, t)$ in spherical polar coordinates $(r, \theta, \phi)$, in the absence of body forces and heat sources, are given by [15]

$$
\begin{gathered}
\sigma_{r r, r}+\frac{1}{r \sin \theta} \sigma_{r \phi, \phi}+\frac{1}{r} \sigma_{r \theta, \theta} \\
+\frac{1}{r}\left[2 \sigma_{r r}-\sigma_{\theta \theta}-\sigma_{\phi \phi}+\sigma_{r \theta} \cot \theta\right]=\rho \ddot{u}_{r} \\
\sigma_{r \phi, r}+\frac{1}{r \sin \theta} \sigma_{\phi \phi, \phi}+\frac{1}{r} \sigma_{\theta \phi, \theta} \\
+\frac{1}{r}\left[3 \sigma_{r \phi}+2 \sigma_{\theta \phi} \cot \theta\right]=\rho \ddot{u}_{\phi} \\
\sigma_{r \theta, r}+\frac{1}{r \sin \theta} \sigma_{\theta \phi, \phi}+\frac{1}{r} \sigma_{\theta \theta, \theta} \\
+\frac{1}{r}\left[3 \sigma_{r \theta}+\left(\sigma_{\theta \theta}-\sigma_{\phi \phi}\right) \cot \theta\right]=\rho \ddot{u}_{\theta} \\
K\left(T,_{r r}+\frac{2}{r} T,,_{r}+\frac{1}{r^{2}}\left\{T,_{\theta \theta}+\cot \theta T,{ }_{\theta}+\frac{1}{\sin ^{2} \theta} T,_{\phi \phi}\right\}\right) \\
-\rho C_{e}\left(\dot{T}+t_{0} \ddot{T}\right)=T_{0} \beta^{*}\left[\dot{e}+t_{0} \delta_{1 k} \ddot{e}\right]
\end{gathered}
$$

where $e=e_{r r}+e_{\theta \theta}+e_{\phi \phi}$

$$
\begin{aligned}
& \sigma_{r r}=\left(\lambda^{*}+2 \mu^{*}\right) e_{r r}+\lambda^{*}\left(e_{\theta \theta}+e_{\phi \phi}\right)-\beta^{*}\left(T+t_{1} \delta_{2 k} \dot{T}\right) \\
& \sigma_{\theta \theta}=\left(\lambda^{*}+2 \mu^{*}\right) e_{\theta \theta}+\lambda^{*}\left(e_{\phi \phi}+e_{r r}\right)-\beta^{*}\left(T+t_{1} \delta_{2 k} \dot{T}\right) \\
& \sigma_{\phi \phi}=\left(\lambda^{*}+2 \mu^{*}\right) e_{\phi \phi}+\lambda^{*}\left(e_{\theta \theta}+e_{r r}\right)-\beta^{*}\left(T+t_{1} \delta_{2 k} \dot{T}\right) \\
& \left(\sigma_{r \theta}, \sigma_{r \phi}, \sigma_{\phi \theta}\right)=2 \mu^{*}\left(e_{r \theta}, e_{r \phi}, e_{\phi \theta}\right) \\
& e_{r r}=\frac{\partial u_{r}}{\partial r}, e_{\theta \theta}=\frac{1}{r} \frac{\partial u_{\theta}}{\partial \theta}+\frac{u_{r}}{r}, \\
& e_{\phi \phi}=\frac{1}{r \sin \theta} \frac{\partial u_{\phi}}{\partial \varphi}+\frac{u_{r}}{r}+\frac{u_{\theta}}{r} \cot \theta \\
& e_{r \phi}=\frac{1}{2}\left[\frac{1}{r \sin \theta} \frac{\partial u_{r}}{\partial \varphi}-\frac{u_{\phi}}{r}+\frac{\partial u_{\phi}}{\partial r}\right] \\
& e_{r \theta}=\frac{1}{2}\left[\frac{1}{r} \frac{\partial u_{r}}{\partial \theta}-\frac{u_{\theta}}{r}+\frac{\partial u_{\theta}}{\partial r}\right] \\
& e_{\theta \phi}=\frac{1}{2}\left[\frac{1}{r} \frac{\partial u_{\phi}}{\partial \theta}-\frac{u_{\phi} \cot \theta}{r}+\frac{1}{r \sin \theta} \frac{\partial u_{\phi}}{\partial r}\right] \text {, } \\
& \lambda^{*}=\lambda\left(1+\alpha_{0} \frac{\partial}{\partial t}\right), \mu^{*}=\mu\left(1+\alpha_{1} \frac{\partial}{\partial t}\right) \\
& \beta^{*}=\beta\left(1+\beta_{0} \frac{\partial}{\partial t}\right), \beta=(3 \lambda+2 \mu) \alpha_{T}, \\
& \beta_{0}=\left(\frac{\left(3 \lambda \alpha_{0}+2 \mu \alpha_{1}\right) \alpha_{T}}{\beta}\right)
\end{aligned}
$$

Here $\sigma_{i j}$ and $e_{i j},(i, j=r, \theta, \phi)$ are the stress and strain components, respectively; $\beta$ is the thermoelastic coupling constant, $\alpha_{0}, \alpha_{1}$ are the viscothermoelastic relaxation times; $\lambda, \mu$ are Lame's parameters; $\alpha_{T}$ is the coefficient of linear thermal expansion; $\rho$ is mass density; $\quad C_{e}$ is the specific heat at constant strain; $K$ is the thermal conductivity; $t_{0}$ and $t_{1}$ are the thermal relaxation times. The quantity $\delta_{i k}, i=1,2$ is Kronecker's delta in which $k=1$ corresponds to Lord-Shulman (LS) and $k=2$ refers to Green-Lindsay (GL) theory of thermoelasticity. The superposed dots represent time differentiation and comma notion is used for spatial derivatives.

\section{Boundary Conditions}

We consider the free vibrations of the sphere which is subjected to stress free, thermally insulated and isothermal conditions and $r=a$ (outer radius) and $r=b$ (inner radius) of the hollow sphere. Mathematically this provides us

$$
\sigma_{r r}=0, \sigma_{r \theta}=0, \sigma_{r \phi}=0, T,_{r}=0
$$

In order to simplify the model, we define following quantities

$$
\begin{aligned}
& r^{\prime}=\frac{r}{R}, t^{\prime}=\frac{c_{1}}{R} t, u_{i}^{\prime}=\frac{u_{i}}{R}, T^{\prime}=\frac{T}{T_{0}} \\
& \sigma_{i j}^{\prime}=\frac{\sigma_{i j}}{\rho c_{1}^{2}}, \alpha_{0}^{\prime}=\frac{c_{1}}{R} \alpha_{0}, \alpha_{1}^{\prime}=\frac{c_{1}}{R} \alpha_{1}, t_{0}^{\prime}=\frac{c_{1}}{R} t_{0}, t_{1}^{\prime}=\frac{c_{1}}{R} t_{1}, \\
& \bar{\beta}=\frac{\beta T_{0}}{\lambda+2 \mu}, \varepsilon_{T}=\frac{T_{0} \beta^{2}}{\rho C_{e}(\lambda+2 \mu)}, \delta^{2}=\frac{c_{2}^{2}}{c_{1}^{2}}, R=\frac{a+b}{2}, \\
& \Omega^{*}=\frac{\omega^{*} R}{c_{1}}, a^{\prime}=\frac{a}{R}, b^{\prime}=\frac{b}{R}, \delta_{0}=\alpha_{0}+2 \delta^{2}\left(\alpha_{1}-\alpha_{0}\right),
\end{aligned}
$$

where $c_{1}=\sqrt{(\lambda+2 \mu) / \rho}, c_{2}=\sqrt{\mu / \rho}$ are longitudinal, shear wave velocities and $\omega^{*}=C_{e}(\lambda+2 \mu) / K$ is characteristic frequency of the medium, $\varepsilon_{T}$ is thermo-mechanical coupling constant. The primes have been suppressed for convenience.

\section{Solution of the Model}

In order to solve the model we introduce the potential functions $\psi, G$ and $w$ defined by [1]

$$
\begin{aligned}
& u_{\theta}=-\frac{1}{\sin \theta} \frac{\partial \psi}{\partial \phi}-\frac{\partial G}{\partial \theta}, \\
& u_{\phi}=\frac{\partial \psi}{\partial \theta}-\frac{1}{\sin \theta} \frac{\partial G}{\partial \phi}, u_{r}=w
\end{aligned}
$$

Upon using the Equation (8) in Equations (1) to (2), we find that $\psi, w, G$ and $T$ satisfy the non-dimensional equations 


$$
\begin{aligned}
& {\left[\left(1+\delta_{0} \frac{\partial}{\partial t}\right)\left(\frac{\partial^{2}}{\partial r^{2}}+\frac{2}{r} \frac{\partial}{\partial r}-\frac{2}{r^{2}}\right)+\delta^{2}\left(1+\alpha_{1} \frac{\partial}{\partial t}\right) \frac{1}{r^{2}} \nabla^{2}-\frac{\partial^{2}}{\partial t^{2}}\right] w} \\
& -\left[\left\{\left(1-\delta^{2}\right)+\left(\delta_{0}-\alpha_{1} \delta^{2}\right) \frac{\partial}{\partial t}\right\} \frac{1}{r} \frac{\partial}{\partial r}-\left\{\left(1+\delta^{2}\right)+\left(\delta_{0}+\alpha_{1} \delta^{2}\right) \frac{\partial}{\partial t}\right\} \frac{1}{r^{2}}\right] \nabla^{2} G-\bar{\beta}\left(1+\beta_{0} \frac{\partial}{\partial t}\right)\left(1+t_{1} \delta_{2 k} \frac{\partial}{\partial t}\right) \frac{\partial T}{\partial r}=0 \\
& -\left[\left\{\left(1-\delta^{2}\right)+\left(\delta_{0}-\alpha_{1} \delta^{2}\right) \frac{\partial}{\partial t} \cdot\right\}\left(\frac{1}{r} \frac{\partial}{\partial r}+\frac{2}{r^{2}}\right)+\delta^{2}\left(1+\alpha_{1} \frac{\partial}{\partial t}\right) \frac{2}{r^{2}}\right] w \\
& +\left[\delta^{2}\left(1+\alpha_{1} \frac{\partial}{\partial t}\right)\left(\frac{\partial^{2}}{\partial r^{2}}+\frac{2}{r} \frac{\partial}{\partial r}\right)+\left(1+\delta_{0} \frac{\partial}{\partial t}\right) \frac{1}{r^{2}} \nabla^{2}-\frac{\partial^{2}}{\partial t^{2}}\right] G+\bar{\beta}\left(1+\beta_{0} \frac{\partial}{\partial t}\right)\left(1+t_{1} \delta_{2 k} \frac{\partial}{\partial t}\right) \frac{T}{r}=0 \\
& {\left[\frac{\partial^{2}}{\partial r^{2}}+\frac{2}{r} \frac{\partial}{\partial r}+\frac{1}{r^{2}} \nabla^{2}-\Omega^{*}\left(\frac{\partial}{\partial t}+t_{0} \frac{\partial^{2}}{\partial t^{2}}\right)\right] T \quad \quad(11) \quad+\frac{\varepsilon_{T} \Omega^{2} \beta_{0}^{*} \tau_{0}^{*} \tau^{\prime} n(n+1)}{\xi} G_{n}+\left(\nabla_{2}^{2}-\frac{\eta^{2}}{\xi^{2}}+\tau_{0}^{*}\right) T_{n}=0} \\
& =\frac{\varepsilon_{T} \Omega^{*}}{\bar{\beta}}\left(\frac{\partial}{\partial t}+t_{0} \delta_{1 k} \frac{\partial^{2}}{\partial t^{2}}\right)\left[\left(\frac{\partial}{\partial r}+\frac{2}{r}\right) w-\frac{1}{r} \nabla^{2} G\right] w_{n} \\
& {\left[\delta^{2}\left(1+\alpha_{1} \frac{\partial}{\partial t}\right)\left(\frac{\partial^{2}}{\partial r^{2}}+\frac{2}{r} \frac{\partial}{\partial r}+\frac{1}{r^{2}} \nabla^{2}\right)-\frac{\partial^{2}}{\partial t^{2}}\right] \psi=0 \quad(12) \quad\left(\nabla_{2}^{2}+\frac{i \Omega^{-1}}{\delta^{2} \alpha_{1}^{*}}-\frac{\eta^{2}}{\xi^{2}}\right) \psi_{n}=0}
\end{aligned}
$$

where $\nabla^{2}=\frac{\partial^{2}}{\partial \theta^{2}}+\cot \theta \frac{\partial}{\partial \theta}+\frac{1}{\sin ^{2} \theta} \frac{\partial^{2}}{\partial \phi^{2}}$

We take wave solution for the displacement functions and temperature change as under

$$
\begin{aligned}
& \psi(r, \theta, \phi, t)=r^{-\frac{1}{2}} \sum_{n=1}^{\infty} \psi_{n}(r) S_{n}^{m}(\theta, \phi) \exp (-i \Omega t) \\
& w(r, \theta, \phi, t)=r^{-\frac{1}{2}} \sum_{n=1}^{\infty} w_{n}(r) S_{n}^{m}(\theta, \phi) \exp (-i \Omega t) \\
& G(r, \theta, \phi, t)=r^{-\frac{1}{2}} \sum_{n=1}^{\infty} G_{n}(r) S_{n}^{m}(\theta, \phi) \exp (-i \Omega t) \\
& T(r, \theta, \phi, t)=r^{-\frac{1}{2}} \sum_{n=1}^{\infty} T_{n}(r) S_{n}^{m}(\theta, \phi) \exp (-i \Omega t)
\end{aligned}
$$

where

$$
\begin{aligned}
& \nabla_{2}^{2}=\frac{1}{\xi} \frac{\mathrm{d}}{\mathrm{d} \xi}\left(\xi \frac{\mathrm{d}}{\mathrm{d} \xi}\right), \xi=r \Omega, b_{1}^{2}=\frac{\left(1-\delta^{2}\right) \delta^{*}}{2 \delta_{0}^{*}}+\frac{\delta^{2} \alpha_{1}^{*}}{\delta_{0}^{*}}+1 \\
& b_{2}^{2}=\frac{1}{4} \frac{\delta^{2} \alpha_{1}^{*}}{\delta_{0}^{*}}+n(n+1), \\
& b_{3}^{2}=\frac{9}{4}+n(n+1) \frac{\delta^{2} \alpha_{1}^{*}}{\delta_{0}^{*}}, \eta^{2}=\left(n+\frac{1}{2}\right)^{2} \\
& \alpha_{0}^{*}=i \Omega^{-1}+\alpha_{0}, \alpha_{1}^{*}=i \Omega^{-1}+\alpha_{1}, \\
& \beta_{0}^{*}=i \Omega^{-1}+\beta_{0}, \delta_{0}^{*}=i \Omega^{-1}+\delta_{0} \\
& \delta^{*}=i \Omega^{-1}+\frac{\delta_{0}-\alpha_{1} \delta^{2}}{1-\delta^{2}}, \tau_{0}=i \Omega^{-1}+t_{0}, \\
& \tau_{0}^{\prime}=i \Omega^{-1}+t_{0} \delta_{1 k}, \tau_{1}=i \Omega^{-1}+t_{1} \delta_{2 k}, \tau_{0}^{*}=\tau_{0} \Omega^{*}, \tau_{0}^{*^{\prime}}=\tau_{0}^{\prime} \Omega^{*}
\end{aligned}
$$

The uncoupling of Equation (17) for $\psi_{n}$ from those of $w_{n}, G_{n}$ and $T_{n}$ indicates the existence of two distinct classes of vibrations in the considered hollow sphere. The solution of Equation (17) for $\psi_{n}$ corresponds to the toroidal modes of vibrations which remains unaffected due to thermal variations and can be discussed in the same manner as was done by Cohen [3] if viscous effects are neglected. The solution of the spherical Bessel Equation (17) is given by

$$
\psi_{n}(\xi)=\sum_{n=1}^{\infty}\left[B_{n 1} J_{\eta}\left(\frac{a^{*}}{\delta} \xi\right)+B_{n 2} Y_{\eta}\left(\frac{a^{*}}{\delta} \xi\right)\right]
$$

$$
+\left[\frac{\delta^{2} \alpha_{1}^{*}}{\delta_{0}^{*}} \nabla_{2}^{2}+\frac{i \Omega^{-1}}{\delta_{0}^{*}}-\frac{b_{2}^{2}}{\xi^{2}}\right] G_{n}-\frac{i \tau_{1} \bar{\beta} \beta_{0}^{*}}{\delta_{0}^{*}} \frac{T_{n}}{\xi}=0
$$

where $\left(a^{*}\right)^{2}=\frac{1}{1-i \Omega \alpha_{1}}, \quad B_{n 1}$ and $B_{n 2}$ are arbitrary constants determined from arbitrary conditions. 


\subsection{Extended Power Series Method}

In order to solve the coupled Equations (14)-(16), we apply Matrix Fröbenius method for the domain of consideration is $b \leq r \leq a$. We take power series of the type

$$
Y_{n}=\sum_{k=0}^{\infty} Z_{k} \xi^{p+k}
$$

where $Y_{n}=\left[\begin{array}{lll}w_{n} & G_{n} & T_{n}\end{array}\right]^{\prime}, Z_{k}=\left[\begin{array}{lll}A_{k} & B_{k} & D_{k}\end{array}\right]^{\prime}$ $p$ is the eigen value and $A_{k}, B_{k}, D_{k}$ are unknowns to be determined.

Substituting the solution (20) in Equations (14)-(16) we get the following matrix equations

$$
\sum_{k=0}^{\infty}\left[H_{1}(p+k) \xi^{-2}+H_{2}(p+k) \xi^{-1}+H\right] \xi^{p+k} Z_{k}=0
$$

where

$$
\begin{aligned}
& H_{1}(p+k)=\left[H_{i j}(p+k)\right]_{3 \times 3}, \\
& H_{2}(p+k)=\left[H_{i j}^{\prime}(p+k)\right]_{3 \times 3}, H=\operatorname{diag}\left(\frac{i \Omega^{-1}}{\delta_{0}^{*}}, \frac{i \Omega^{-1}}{\delta_{0}^{*}}, \tau_{0}^{* \prime}\right)
\end{aligned}
$$

The non zero elements of the matrices $H_{1}(p+k)$ and $H_{2}(p+k)$ are given by

$$
\begin{aligned}
& H_{11}(p+k)=\left((p+k)^{2}-b_{3}^{2}\right), \\
& H_{12}(p+k)=n(n+1)\left(c_{3}^{*}\left(p+k-b_{1}^{2}\right)\right. \\
& H_{21}(p+k)=-\left(c_{3}^{*}(p+k)+b_{1}^{2}\right), \\
& H_{22}(p+k)=\left(c_{1}^{*}(p+k)^{2}-b_{2}^{2}\right) \\
& H_{33}(p+k)=\left((p+k)^{2}-\eta^{2}\right), \\
& H_{13}^{\prime}(p+k)=c_{2}^{*}\left(p+k-\frac{1}{2}\right), H_{23}^{\prime}(p+k)=-c_{2}^{*} \\
& H_{31}^{\prime}(p+k)=\varepsilon_{T} \frac{\Omega \tau_{0}^{* \prime}}{\bar{\beta}}\left(p+k+\frac{3}{2}\right), \\
& H_{32}^{\prime}(p+k)=\varepsilon_{T} \frac{\Omega \tau_{0}^{* \prime}}{\bar{\beta}} n(n+1) \\
& \text { where } c_{1}^{*}=\frac{\delta^{2} \alpha_{1}^{*}}{\delta_{0}^{*}}, c_{2}^{*}=\frac{i \tau_{1} \beta_{0}^{*} \bar{\beta}}{\delta_{0}^{*}}, c_{3}^{*}=\frac{\left(1-\delta^{2}\right) \delta^{*}}{\delta_{0}^{*}} .
\end{aligned}
$$

Equating to zero the coefficients of lowest powers of $\xi$ (i.e. $\xi^{p-2}=0$ ) in Equation (21), we obtain

$$
H(p) Z_{0}=0
$$

where

$$
H(p)=\left[\begin{array}{ccc}
\left(p^{2}-b_{3}^{2}\right) & n(n+1)\left(c_{3}^{*} p-b_{1}^{2}\right) & 0 \\
-\left(c_{3}^{*} p+b_{1}^{2}\right) & \left(c_{1}^{*} p^{2}-b_{2}^{2}\right) & 0 \\
0 & 0 & \left(p^{2}-\eta^{2}\right)
\end{array}\right],
$$$$
Z_{0}=\left[\begin{array}{lll}
A_{0} & B_{0} & D_{0}
\end{array}\right]
$$

The requirement for the existence of non-trivial solution of Equation (24) leads to the following two indicial equations

$$
p^{4}-A p^{2}+C=0, p^{2}=\left(n+\frac{1}{2}\right)^{2}
$$

where $A=\left(\left(b_{2}^{2}+c_{1}^{*} b_{3}^{2}\right)-n(n+1)\left(c_{3}^{*}\right)^{2}\right) / c_{1}^{*}$,

$C=\left(b_{2}^{2} b_{3}^{2}-n(n+1) b_{1}^{4}\right) / c_{1}^{*}$

The roots of Equation (26) are of the type $p= \pm p(i=$ $1,2,3)$

where

$$
p_{1}=\sqrt{\left(\frac{A+\sqrt{A^{2}-4 C}}{2}\right)}, p_{2}=\sqrt{\left(\frac{A-\sqrt{A^{2}-4 C}}{2}\right)}, p_{3}=\eta
$$

We designate the roots of Equation (26) as $p_{i}(i=1$ to 6$)$ with $p_{4}=-p_{1}, p_{5}=-p_{2}, p_{6}=-p_{3}$. Obviously $p_{3}, p_{6}$ are real and $p_{1}, p_{2}, p_{4}, p_{5}$ may be complex in general. If $p_{i},(i=1,2,4,5)$ are complex, then leading terms in the complex series solution (24) are of the type

$$
\begin{aligned}
& {\left[\begin{array}{lll}
A_{0} & B_{0} & D_{0}
\end{array}\right] \xi^{p}=Z_{0} \xi^{p_{R}+i_{I}} } \\
= & Z_{0} \xi^{p_{R}}\left[\cos \left(p_{I} \log \xi\right)+i \sin \left(p_{I} \log \xi\right)\right]
\end{aligned}
$$

In order to obtain two independent real solutions, it is sufficient to use any one of the complex root and taking its real and imaginary parts see Neuringer [10]. Moreover, the treatment of complex case is unlike that of the real roots with the advantage that the differential equation is required to be solved only once in the former case rather than twice in the later one. For the choice of roots of the indicial equations, the system of Equations (24) leads to following eigen vectors

$$
\begin{aligned}
& Z_{0}\left(p_{1}\right)=\left[\begin{array}{lll}
1 & Q_{B}\left(p_{1}\right) & 0
\end{array}\right]^{\prime} L_{0}, Z_{0}\left(p_{2}\right)
\end{aligned}
$$

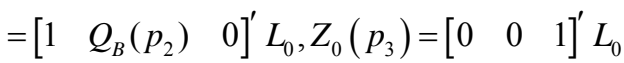

$$
\begin{aligned}
& Z_{0}\left(p_{4}\right)=\left[\begin{array}{lll}
1 & Q_{B}\left(-p_{1}\right) & 0
\end{array}\right]^{\prime} L_{0}, Z_{0}\left(p_{5}\right)
\end{aligned}
$$

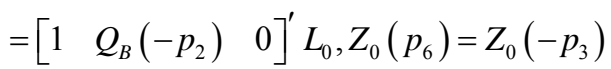

where

$$
\begin{gathered}
Q_{B}\left(p_{j}\right)=-\frac{\left(p_{j}^{2}-b_{3}^{2}\right)}{n(n+1)\left(c_{3}^{*} p_{j}-b_{1}^{2}\right)}=\frac{c_{3}^{*} p_{j}+b_{1}^{2}}{c_{1}^{*} p_{j}^{2}-b_{2}^{2}},(j=1,2) \\
Q_{B}\left(-p_{j}\right)=\frac{\left(p_{j}^{2}-b_{3}^{2}\right)}{n(n+1)\left(c_{3}^{*} p_{j}+b_{1}^{2}\right)}=\frac{-c_{3}^{*} p_{j}+b_{1}^{2}}{c_{1}^{*} p_{j}^{2}-b_{2}^{2}},(j=1,2)
\end{gathered}
$$

and $L_{0}$ is a constant. This suggest us to have 


$$
\begin{aligned}
& A_{0}\left(p_{j}\right)=\left\{\begin{array}{l}
1, j=1,2 \\
0, j=3
\end{array}, B_{0}\left(p_{j}\right)=\left\{\begin{array}{l}
Q_{B}\left(p_{j}\right), j=1,2 \\
0, j=3
\end{array},\right.\right. \\
& D_{0}\left(p_{j}\right)=\left\{\begin{array}{l}
0, j=1,2 \\
1, j=3
\end{array}\right.
\end{aligned}
$$

where $A_{0}\left(p_{j}\right), B_{0}\left(p_{j}\right), D_{0}\left(p_{j}\right) ; j=4,5,6$ can be written from (31) by replacing $p_{j}$ with $-p_{j},(j=1,2,3)$ and again equating to zero the coefficients of next lowest degree term $\xi^{p-1}$, which corresponds to $k=1$, and using Equations (21) we obtain:

$$
H_{1}\left(p_{j}+1\right) Z_{1}+H_{2}\left(p_{j}\right) Z_{0}=0,(j=1,2,3)
$$

Clearly $H_{1}\left(p_{j}+1\right)$ is non-singular for each $p_{j}$, therefore we have:

$$
Z_{1}=-\left(H_{1}\left(p_{j}+1\right)\right)^{-1} H_{2}\left(p_{j}\right) Z_{0}=D_{1}^{*} Z_{0}
$$

where

$$
\begin{aligned}
& Z_{1}=\left[\begin{array}{lll}
A_{1} & B_{1} & D_{1}
\end{array}\right]^{\prime}, \\
& D_{1}^{*}=-\left(H_{1}\left(p_{j}+1\right)\right)^{-1} H_{2}\left(p_{j}\right)=\left[\begin{array}{ccc}
0 & 0 & L_{13} \\
0 & 0 & L_{23} \\
L_{31} & L_{32} & 0
\end{array}\right]
\end{aligned}
$$

$L_{i j},(i, j=1,2,3)$ are given by Equations (A.1) to (A.4) of Appendix. Here the matrices $H_{1}\left(p_{j}+1\right)$ and $H_{2}\left(p_{j}\right)$ can be written from the Equations (22) and (23) by set- ting $k=1$. Now equating the coefficients of powers of $\xi^{p+k}$ equal to zero, we obtain following recurrence rela- tion:

$$
H_{1}\left(p_{j}+k+2\right) Z_{k+2}+H_{2}\left(p_{j}+k+1\right) Z_{k+1}+H Z_{k}=0(35)
$$

where the matrices $H_{1}, H_{2}$ and $H$ are defined in Equation (22) and (23). This implies that

$$
\begin{aligned}
Z_{k+2}= & -\left(H_{1}\left(p_{j}+k+2\right)\right)^{-1} \\
& \times\left[H_{2}\left(p_{j}+k+1\right) Z_{k+1}+H Z_{k}\right]
\end{aligned}
$$

Now putting $k=0,1,2,3 \cdots$ successively, and simplifying we get

$$
\begin{aligned}
& Z_{k+2}=-\left(H_{1}\left(p_{j}+k+2\right)\right)^{-1} \\
& \times\left[H_{2}\left(p_{j}+k+1\right) D_{k+1}^{*}+H D_{k}^{*}\right] Z_{0}=D_{k+2}^{*} Z_{0} \\
& \{w, G, T\}(r, \theta, \phi, t)=(r)^{-\frac{1}{2}} \sum_{n=0}^{\infty} \sum_{j=1}^{6} \sum_{k=0}^{\infty} C_{n j k}\left\{A_{k}\left(p_{j}\right), B_{k}\left(p_{j}\right), D_{k}\left(p_{j}\right)\right\}(r \Omega)^{p_{j}+k} P_{n}^{m}(\cos \theta) e^{-i(m \phi+\Omega t)} \\
& \psi(r, \theta, \phi, t)=(r)^{-\frac{1}{2}}\left[\sum_{n=1}^{\infty} B_{n 1} J_{\eta}\left(\frac{a^{*}}{\delta} r \Omega\right)+\sum_{n=1}^{\infty} B_{n 2} Y_{\eta}\left(\frac{a^{*}}{\delta} r \Omega\right)\right] P_{n}^{m}(\cos \theta) e^{-i(m \phi+\Omega t)}
\end{aligned}
$$


where $\left\{A_{k}\left(p_{j}\right), B_{k}\left(p_{j}\right), D_{k}\left(p_{j}\right)\right\}$ are eigenvectors shown in Appendix (A.15) corresponding to the eigenvalues $p_{j}$. The unknowns $B_{n 1}, B_{n 2}$ and
$C_{n j k},(j=1,2,3,4,5,6)$ can be evaluated by four boundary conditions of hollow sphere. Displacements and stresses are obtained as

$$
\begin{aligned}
& u_{r}=(r)^{-\frac{1}{2}} \sum_{n=0}^{\infty}\left[\sum_{j=1}^{6} \sum_{k=0}^{\infty} C_{n j k} A_{k}\left(p_{j}\right)(r \Omega)^{p_{j}+k}\right] P_{n}^{m}(\cos \theta) \exp [-i(m \phi+\Omega t)] \\
& u_{\theta}=(r)^{-\frac{1}{2}}\left[\frac{i m}{\sin \theta} \sum_{n=1}^{\infty} B_{n 1} \bar{F} P+\sin \theta\left(\sum_{n=0}^{\infty} \sum_{j=1}^{6} \sum_{k=1}^{\infty} C_{n j k} B_{k}\left(p_{j}\right)(r \Omega)^{p_{j}+k}\right) P^{\prime}\right] F^{*} \\
& u_{\phi}=(r)^{-\frac{1}{2}}\left[\frac{i m}{\sin \theta}\left(\sum_{n=0}^{\infty} \sum_{j=1}^{6} \sum_{k=1}^{\infty} C_{n j k} B_{k}\left(p_{j}\right)(r \Omega)^{p_{j}+k}\right) P-\sin \theta \sum_{n=1}^{\infty} B_{n 1} \bar{F} P^{\prime}\right] F^{*} \\
& \sigma_{r r}=(r)^{-\frac{1}{2}}\left[\sum_{j=1}^{6}\left\{\sum_{n=0}^{\infty} C_{n j 0}\left(p_{j}+2 A_{0}^{*}-\frac{1}{2}\right) A_{0}\left(p_{j}\right)+\sum_{n=1}^{\infty} C_{n j 0} n(n+1) A_{0}^{*} B_{0}\left(p_{j}\right)\right\}(r \Omega)^{p_{j}-1}\right. \\
&\left.+\sum_{k=0}^{\infty} \sum_{j=1}^{6}\left\{\sum_{n=1}^{\infty} C_{n j k} n(n+1) A_{0}^{*} B_{k+1}\left(p_{j}\right)+\sum_{n=0}^{\infty} C_{n j k}\left(p_{j}+k+2 A_{0}^{*}+\frac{1}{2}\right) A_{k+1}\left(p_{j}\right)+C_{2}^{*} D_{k}\left(p_{j}\right)\right\}(r \Omega)^{p_{j}+k}\right] P F^{*} \\
& \sigma_{r \theta}=-(r)^{-\frac{1}{2}}\left[\sum_{j=1}^{6}\left(\left\{\sum_{n=0}^{\infty} C_{n j 0} A_{0}\left(p_{j}\right)+\sum_{n=1}^{\infty} C_{n j 0}\left(\frac{3}{2}-p_{j}\right) B_{0}\left(p_{j}\right)\right\}\right) \bar{P} P^{\prime}(r \Omega)^{p_{j}-1}\right. \\
&\left.+\sum_{k=0}^{\infty} \sum_{j=1}^{6}\left\{\sum_{n=0}^{\infty} C_{n j k} A_{k+1}\left(p_{j}\right)+\sum_{n=0}^{\infty} C_{n j k}\left(\frac{1}{2}-p_{j}-k\right) B_{k+1}\left(p_{j}\right)\right\} \bar{P} P^{\prime}(r \Omega)^{p_{j}+k}+\sum_{n=1}^{\infty} i m F \frac{P}{\bar{P}}\right] F^{*} \\
&\left.\quad+\sum_{k=0}^{\infty} \sum_{j=1}^{6}\left\{\sum_{n=0}^{\infty} C_{n j k} A_{k+1}\left(p_{j}\right)+\sum_{n=0}^{\infty} C_{n j k}\left(\frac{1}{2}-p_{j}-k\right) B_{k+1}\left(p_{j}\right)\right\} i m \frac{P}{\bar{P}}(r \Omega)^{p_{j}+k}-\sum_{n=1}^{\infty} F \bar{P} P^{\prime}\right] F^{*} \\
& \sigma_{r \phi}=-(r)^{-\frac{1}{2}}\left[\sum_{j=1}^{6}\left\{\sum_{n=0}^{\infty} C_{n j 0} A_{0}\left(p_{j}\right)+\sum_{n=1}^{\infty} C_{n j 0}\left(\frac{3}{2}-p_{j}\right) B_{0}\left(p_{j}\right)\right\} i m \frac{P}{\bar{P}}(r \Omega)^{p_{j}-1}\right.
\end{aligned}
$$

where $\bar{F}=\sum_{n=1}^{\infty} B_{n 1} J_{\eta}\left(\frac{a^{*}}{\delta} r \Omega\right)+\sum_{n=1}^{\infty} B_{n 2} Y_{\eta}\left(\frac{a^{*}}{\delta} r \Omega\right)$

$$
\begin{aligned}
F= & B_{n 1}\left[\left(\eta-\frac{3}{2}\right) J_{\eta}\left(\frac{a^{*}}{\delta} r \Omega\right)-\frac{a^{*}}{\delta} r \Omega J_{\eta+1}\left(\frac{a^{*}}{\delta} r \Omega\right)\right] \\
& +B_{n 2}\left[\left(\eta-\frac{3}{2}\right) Y_{\eta}\left(\frac{a^{*}}{\delta} r \Omega\right)-\frac{a^{*}}{\delta} r \Omega Y_{\eta+1}\left(\frac{a^{*}}{\delta} r \Omega\right)\right] \\
F^{*} & =\exp [-i(m \phi+\Omega t)], P=P_{n}^{m}(\cos \theta) \\
\bar{P} & =\sin \theta, P^{\prime}=\frac{\partial P_{n}^{m}(\cos \theta)}{\partial(\cos \theta)}, A_{0}^{*}=\frac{\left(1-2 \delta^{2}\right) \alpha_{0}^{*}}{\delta_{0}^{*}}
\end{aligned}
$$

\section{Secular Dispersion Relations}

On applying the boundary conditions on Equations (6) we obtain a system of eight homogeneous linear algebraic equations which will have a nontrivial solution if and only if the determinant of the coefficients $B_{n 1}, B_{n 2}$ and $C_{n j k},(j=1,2,3,4,5,6)$ vanishes. This requirement of nontrivial solution leads to a determinant equations for thermally insulated hollow sphere which further splits into two different classes of vibrations discussed below.

\subsection{For Stress Free Boundary Conditions}

Case 1: For $k=0, n>0$, the secular equations are given as

$$
\begin{gathered}
\operatorname{det}\left(d_{i j}\right)=0,(i, j=1,2,3,4,5,6) \\
d_{77} d_{88}-d_{87} d_{78}=0 \\
\left(1-\mu^{2}\right) \frac{\mathrm{d} P_{n}^{m}(\mu)}{\mathrm{d} \mu}-m P_{n}^{m}(\mu)=0,
\end{gathered}
$$

where $\mu=\cos \theta$

$$
\begin{aligned}
d_{11}= & {\left[n(n+1) A_{0}^{*} Q_{B}\left(p_{1}\right)+\left(p_{1}+2 A_{0}^{*}+\frac{1}{2}\right)\right] } \\
& \times A_{0}\left(p_{1}\right)(a \Omega)^{p_{1}} \\
d_{31}= & \left\{\begin{array}{l}
\left(p_{1}+k+\frac{1}{2}\right) D_{k+1}\left(p_{1}\right)(a \Omega)^{p_{1}+k} ; \text { thermally insulated } \\
D_{k}\left(p_{1}\right)(a \Omega)^{p_{1}+k} ; \text { isothermal }
\end{array}\right.
\end{aligned}
$$




$$
\begin{aligned}
& d_{51}=\left(\frac{3}{2}-p_{1}\right) Q_{B}\left(p_{1}\right) A_{0}\left(p_{1}\right)(a \Omega)^{p_{1}} \\
& d_{77}=\left[\left(\eta-\frac{3}{2}\right) J_{\eta}\left(\frac{a^{*}}{\delta} a \Omega\right)-\frac{a^{*}}{\delta} a \Omega J_{\eta+1}\left(\frac{a^{*}}{\delta} a \Omega\right)\right]
\end{aligned}
$$

Here the elements $\operatorname{det}\left(d_{i j}\right),(i, j=1,2,3,4,5,6)$ of Equation (40) can be written from $\operatorname{det}\left(d_{i j}\right),(i=1,3,5)$ by replacing $p_{1}$ with $p_{j}(j=2,4,6)$ and the elements $\operatorname{det}\left(d_{i j}\right),(i=2,4,6$ and $j=1-6)$ are obtained by replacing $a$ with $b$. The element $d_{78}$ can be obtained by replacing Bessel's function of first kind $J_{\eta}$ with that of second kind $Y_{\eta}$ in Equation (43.4) and the elements $d_{87}$ and $d_{88}$ can be obtained from $d_{77}$ and $d_{78}$ by replacing $a$ with $b$, respectively.

Case 2: For $k>0, n=0$ the secular equations are obtained as

$$
\operatorname{det}\left(d_{i j}^{\prime}\right)=0,(i, j=1,2,3,4,5,6),
$$

where

$$
\begin{aligned}
& d_{11}^{\prime}=\left[\left(p_{1}+k+2 A_{0}^{*}+\frac{1}{2}\right) A_{k+1}\left(p_{1}\right)+c_{2} D_{k}\left(p_{1}\right)\right](a \Omega)^{p_{1}+k} \\
& d_{31}^{\prime}=\left\{\begin{array}{l}
\left(p_{1}+k+\frac{1}{2}\right) D_{k+1}\left(p_{1}\right)(a \Omega)^{p_{1}+k} ; \text { thermally insulated } \\
D_{k}\left(p_{1}\right)(a \Omega)^{p_{1}+k} ; \text { isothermal }
\end{array}\right. \\
& d_{51}^{\prime}=A_{k+1}\left(p_{1}\right)(a \Omega)^{p_{1}+k}
\end{aligned}
$$

The elements $\operatorname{det}\left(d_{i j}^{\prime}\right),(i, j=1,2,3,4,5,6)$ of Equation (44) can be obtained by just replacing $p_{1}$ in $\operatorname{det}\left(d_{i j}^{\prime}\right),(i=1,3,5)$ with $p_{j},(j=2,3,4,5,6)$ while $\operatorname{det}\left(d_{i j}^{\prime}\right),(i=2,4,6)$ are obtained by replacing $a$ in $\operatorname{det}\left(d_{i j}^{\prime}\right),(i=1,3,5)$ with $b$.

Case 3: For $k>0, n>0$, the secular equations are given as

$$
\operatorname{det}\left(\bar{d}_{i j}^{\prime}\right)=0,(i, j=1,2,3,4,5,6)
$$

where

$$
\begin{aligned}
& \bar{d}_{11}^{\prime}= {\left[n(n+1) A_{0}^{*} B_{k+1}\left(p_{1}\right)+\left(p_{1}+k+\frac{1}{2}+2 A_{0}^{*}\right)\right.} \\
&\left.\times A_{k+1}\left(p_{1}\right)+c_{2} D_{k}\left(p_{1}\right)\right](a \Omega)^{p_{1}+k} \\
& \bar{d}_{31}^{\prime}=\left\{\begin{array}{l}
\left(p_{1}+k+\frac{1}{2}\right) D_{k+1}\left(p_{1}\right)(a \Omega)^{p_{1}+k} ; \text { thermally insulated } \\
D_{k}\left(p_{1}\right)(a \Omega)^{p_{1}+k} ; \text { isothermal }
\end{array}\right. \\
& \bar{d}_{51}^{\prime}=\left[A_{k+1}\left(p_{1}\right)+\left(\frac{1}{2}-p_{1}-k\right) B_{k+1}\left(p_{1}\right)\right](a \Omega)^{p_{1}+k}
\end{aligned}
$$

The elements of $\operatorname{det}\left(\bar{d}_{i j}^{\prime}\right),(i, j=1,2,3,4,5,6)$ of determinant equation (45) can be obtained by just replacing $p_{1}$ in $\operatorname{det}\left(\bar{d}_{i j}^{\prime}\right),(i=1,3,5)$ with $p_{j},(j=2,3,4,5,6)$

while $\operatorname{det}\left(\bar{d}_{i j}^{\prime}\right),(i=2,4,6)$ are obtained by replacing $a$ in $\operatorname{det}\left(\bar{d}_{i j}^{\prime}\right)=0,(i=1,3,5)$ with $b$.

\subsection{First Class Vibrations}

The vibrations of first class correspond to the solution of Equation (38.2) of potential function $\psi$ and hence are given by Equation (41). After lengthy but straight forward calculations, the characteristic Equation (41) can be simplified by using asymptotic Expansion [3] for functions as

$$
\frac{\tan \left(\left(a^{*} / \delta\right) \Omega h\right)}{\left(a^{*} / \delta\right) \Omega h} \cong \frac{4 \eta^{2}+15}{8 a b \Omega^{2}-4 \eta^{2}+33}
$$

where $h=a-b, R=\frac{a+b}{2}$ is the mean radius.

In the limiting case of the thickness $h$ tending to zero, we obtain from Equation (46)

$$
R^{2} \Omega^{2} \cong \eta^{2}-\frac{9}{4}
$$

These Equations (46) and (47) are of similar type reported by Cohen et al. [3] but completely agreement in the absence of viscous effect.

If we take $\eta=n+\frac{1}{2}$ the Equation (46) reduces to

$$
\begin{aligned}
& \frac{\tan \left(\left(a^{*} / \delta\right) \Omega h\right)}{\left(a^{*} / \delta\right) \Omega h} \cong \frac{2}{a b \Omega^{2}+4} \text { for } n=0 \\
& \frac{\tan \left(\left(a^{*} / \delta\right) \Omega h\right)}{\left(a^{*} / \delta\right) \Omega h} \cong \frac{3}{a b \Omega^{2}+3} \text { for } n=1 \\
& \frac{\tan \left(\left(a^{*} / \delta\right) \Omega h\right)}{\left(a^{*} / \delta\right) \Omega h} \cong \frac{\left(n+\frac{1}{2}\right)^{2}+\frac{15}{4}}{2 a b \Omega^{2}-\left(\left(n+\frac{1}{2}\right)^{2}-\frac{33}{4}\right)} \text { for } n>1
\end{aligned}
$$

\subsection{Second Class Vibrations}

The secular Equations (40), (44) and (45) govern the second class vibrations called spheroidal vibrations (S-modes) for Case 1. $k=0, n>0$, Case 2. $k>0, n=0$, and Case 3. $k>0, n>0$, Stress free conditions respectively.

\subsection{Thermo-Elastic Hollow Sphere}

If we ignore the viscous effect $\left(\alpha_{0}=0=\alpha_{1}\right)$, then the present analysis reduces to that of generalized thermoelastic hollow sphere. In case the thermal relaxation time 
is zero $\left(t_{0}=0=t_{1}\right)$, the above results reduce to those which govern the vibrations of coupled thermoelastic hollow sphere.

\subsection{Viscoelastic Hollow Sphere}

If thermal equilibrium is assumed to be established then $\left(\varepsilon_{T}=0=T, t_{0}=0=t_{1}\right)$, and present analysis reduces to one which governs the spheroidal and toroidal vibrations of a viscoelastic hollow sphere.

\subsection{Elastic Sphere}

When in addition to establishment of thermal equilibrium, the viscous effect in solid is ignored so that

$\varepsilon_{T}=0=T, t_{0}=0=t_{1}, \alpha_{0}=0=\alpha_{1}$, then the above analysis completely reduces to one that governs the spheroidal and toroidal vibrations of an elastic hollow sphere. In this case the results are observed to be in agreement with Cohen [3].

\section{Numerical Results and Discussion}

In order to illustrate the analytical developments, we propose some numerical calculations to compute lowest frequency of S-modes in the hollow sphere made of copper material. The numerical computations have been carried out for spheroidal modes of vibrations for $k>0, n>0$ by using fixed point iteration numerical technique with the help of MATLAB software tools for thickness to mean radius ratio. Due to the presence of dissipation term in heat conduction Equation (2), the secular equations are, in general, complex transcendental equations and hence provide us complex values of the frequency $\Omega$ and hence of $\omega$. If we write $\omega=\omega_{R}+i \omega_{I}$, then the lowest frequency and dissipation factor are given by $\hat{\Omega}=\operatorname{Re}(\Omega / \delta)=R \omega_{R} / c_{2}$ and $D=\operatorname{Im}(\Omega / \delta)=R \omega_{1} / c_{2}$, for fixed values of $n$ and $k$. The numerical computations have been done by taking sufficient number of values of the Fröbenius parameter $k$ in order to obtain the converged values of lowest frequency $(\hat{\Omega})$ and dissipation factor $(D)$ of S-modes. The computer simulated lowest frequency, dissipation factor, displacements; stresses and temperature change have been presented in Figures 1 to 10 for viscothermoelastic (VTE), thermoelastic (TE), viscoelastic (VE), elastic (E) materials of hollow sphere. The material copper has been taken for the computation purpose as whose physical data [14] is given as

$$
\begin{aligned}
& \lambda=8.2 \times 10^{10} \mathrm{Nm}^{-2}, \mu=4.2 \times 10^{10} \mathrm{Nm}^{-2}, \\
& \rho=8.950 \times 10^{10} \mathrm{~kg} \cdot \mathrm{m}^{-3}, \varepsilon_{T}=0.00265, \alpha_{T}=1.0 \times 10^{-8} / \mathrm{K}, \\
& K=1.13 \times 10^{2} \mathrm{Cal} \cdot \mathrm{m}^{-1} \cdot \mathrm{s}^{-1} \cdot \mathrm{K}^{-1}, \\
& \alpha_{0}=\alpha_{1}=6.8831 \times 10^{-13} \mathrm{~s}, \omega^{*}=1.11 \times 10^{11} \mathrm{~s}^{-1}, T_{0}=300 \mathrm{~K} .
\end{aligned}
$$

The variations of lowest frequency $\hat{\Omega}$ and dissipa- tion factor in a stress-free and thermally insulated hollow sphere of copper material versus degree of harmonics $(n)$ have been plotted in Figures $\mathbf{1}$ and $\mathbf{2}$ at different values of thickness to mean radial ratio $\left(t^{*}=0.2,0.4,0.6,1.0\right)$, where $t^{*}=h / R, h=a-b, R=(a+b) / 2$. It is concluded from Figure 1 that the lowest frequency increases with increase in degree of harmonics. It can be inferred from Figure 2 that with increase in degree of harmonics the dissipation of vibration modes go on increasing. Figure 3 the lowest frequency of toroidal vibrations has been plotted versus degree of harmonics $(n)$ for different values of thickness to mean radial ratio $\left(t^{*}=0.2,0.4,0.6,1\right)$. From Figure 3 it is revealed that with increase in the degree of harmonics the frequency of vibrations go on increasing. The trends of the profiles in Figure 3 are similar to that as reported in Ding and Chen [17]. But the dissipation factor of toroidal vibrations is very low (of the order $10^{-10}$ )

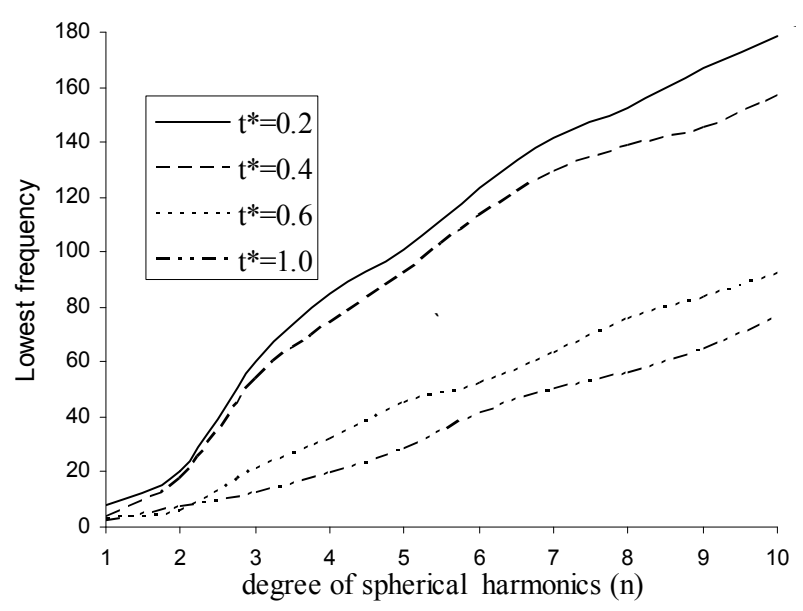

Figure 1. Lowest frequency $(\hat{\boldsymbol{\Omega}})$ of spheroidal vibrations versus degree of harmonics $(n)$ in (VTE) hollow sphere.

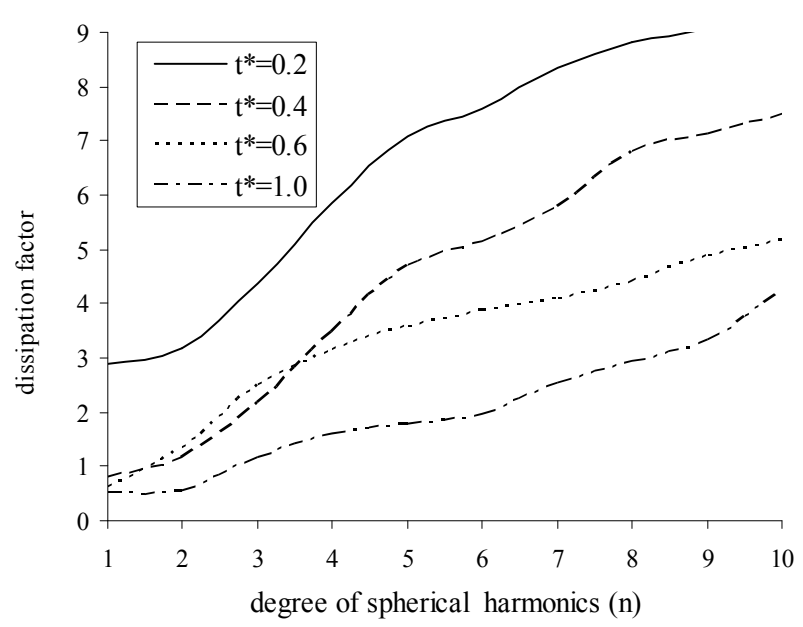

Figure 2. Dissipation $(D)$ of spheroidal vibrations versus degree of harmonics $(n)$ in (VTE) hollow sphere. 


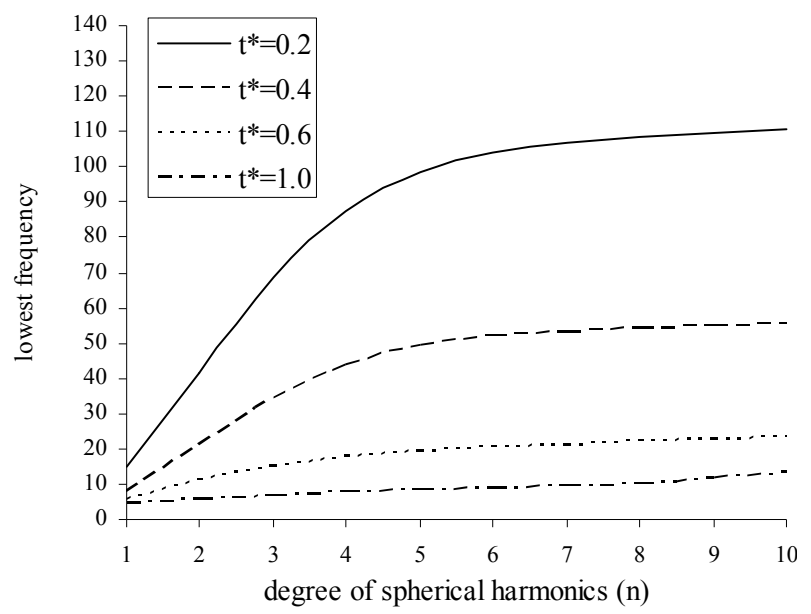

Figure 3. Lowest frequency $(\hat{\mathbf{\Omega}})$ of Toroidal vibrations versus degree of harmonics $(n)$ in (VTE) hollow sphere

in the instant case which is negligible. From the trends of variations of lowest frequency and dissipation factor of $\mathrm{S}$-mode, it is noticed that the thermal variations, thermal relaxation time and viscous nature of the material significantly affect the characteristics and behavior of spherecal vibrations and their magnitudes in contrast to that of toroidal modes which are only affected due to viscosity but not by the temperature variations as expected.

In Figures 4 to 7 , the variations of temperature change (T) and stresses $\left(\sigma_{r r}, \sigma_{r \theta}, \sigma_{r \varphi}\right)$ versus $\eta^{*}=((r-b) / h)$ i.e. $0 \leq((r-b) / h) \leq 1$ (difference in radius and inner radius to thickness) for the modes $(0,0),(1,0)$ and $(1,1)$ have been plotted in case of stress free and thermally insulated surface of the (VTE) sphere. Figure 4 revealed that the magnitude of temperature change is though meager, but decreases with increasing values of $\left(\eta^{*}\right)$ from its maximum value at $\eta^{*}=0$ to become steady and stable at the $\eta^{*}=1$ in case of all the modes. It is inferred from Figure 5 that the variations of radial stress $\left(\sigma_{r r}\right)$ for modes $(m, n):(1,0),(1,1)$ initially increases and die out with increase in values of $\left(\eta^{*}\right)$. But for the mode $(0,0)$, the stress is of compressive nature and its magnitude vanishes with increasing values of $\left(\eta^{*}\right)$. It is noticed from Figure 6 that the meridian stress $\left(\sigma_{r \theta}\right)$ of vibration modes $(m, n):(1,0),(1,1)$ has compressive nature and it dies out with increasing values of $\left(\eta^{*}\right)$. However the mode $(0,0)$ has maximum variations of this quantity and its vibrations die out with increasing values of $\left(\eta^{*}\right)$. Figure 7 shows that the variation of stress for mode $(1,1)$. It has maximum value and its magnitude goes on decreasing with increasing values of $\eta^{*}$ to ultimately die out.

Figures 8 to $\mathbf{1 0}$ represent the variations of displacements $\left(u_{r}, u_{\theta}, u_{\varphi}\right)$ versus $\left(\eta^{*}\right)$ for the modes

$(m, n):(0,0),(1,0)$ and $(1,1)$. Figure 8 revealed that the variations of radial displacement decrease from their maximum variation $\eta^{*}=0$ with increasing values of $\left(\eta^{*}\right)$ to become stable at $\eta^{*}=1$ for all modes of vibrations. Figures 9 and $\mathbf{1 0}$ show the variations of displacements $u_{\theta}$ and $u_{\phi}$ versus $\left(\eta^{*}\right)$. The profiles of

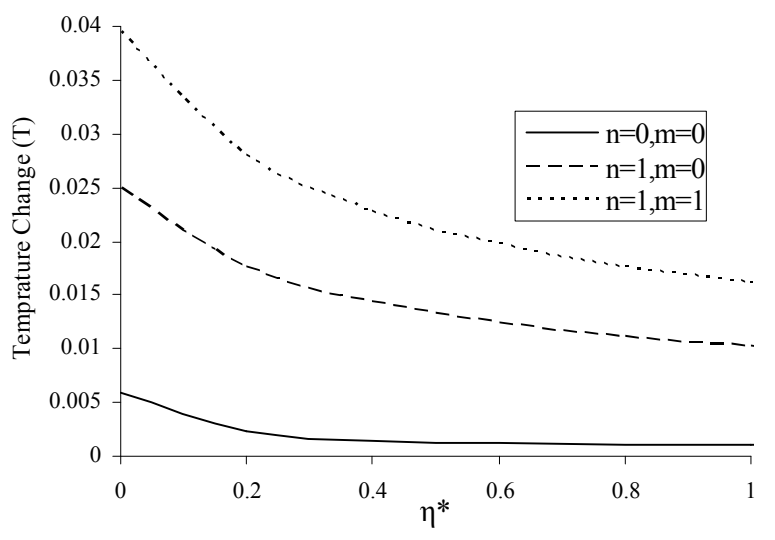

Figure 4. Variation of temperature change $(T)$ versus $\left(\eta^{*}\right)$ of hollow sphere.

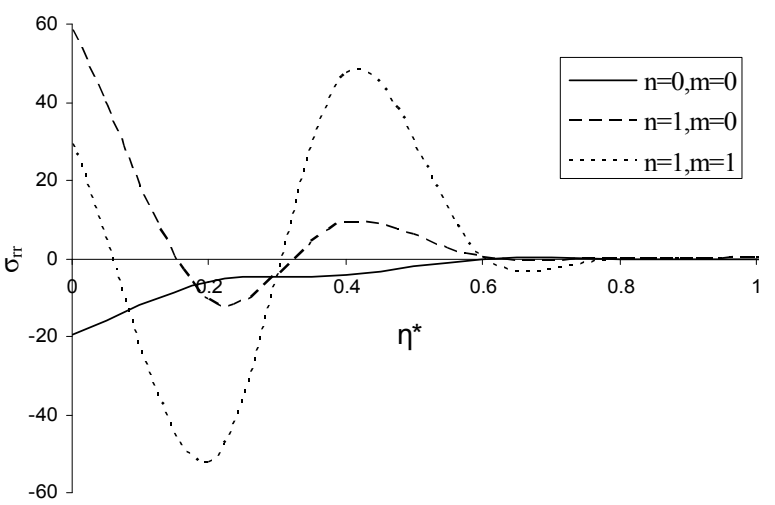

Figure 5. Variation of stress $\left(\sigma_{r r}\right)$ verses $\left(\eta^{*}\right)$ of hollow sphere.

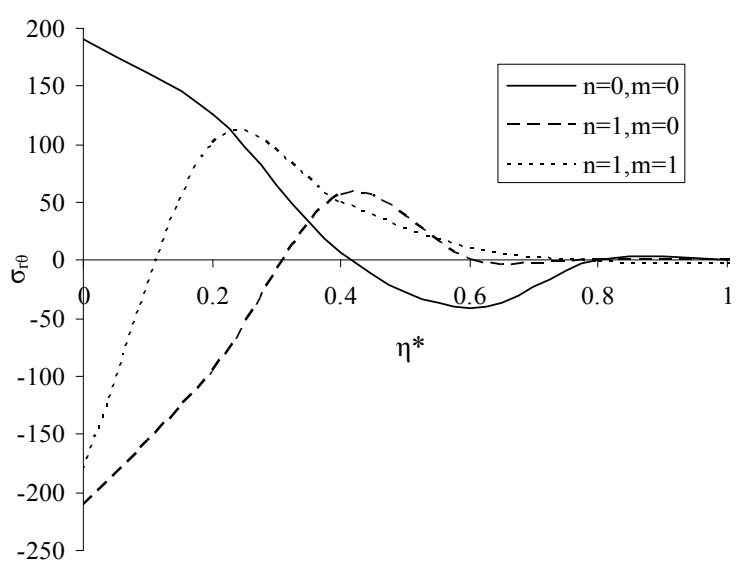

Figure 6. Variation of stress $\left(\sigma_{r \theta}\right)$ verses $\left(\eta^{*}\right)$ of hollow sphere. 


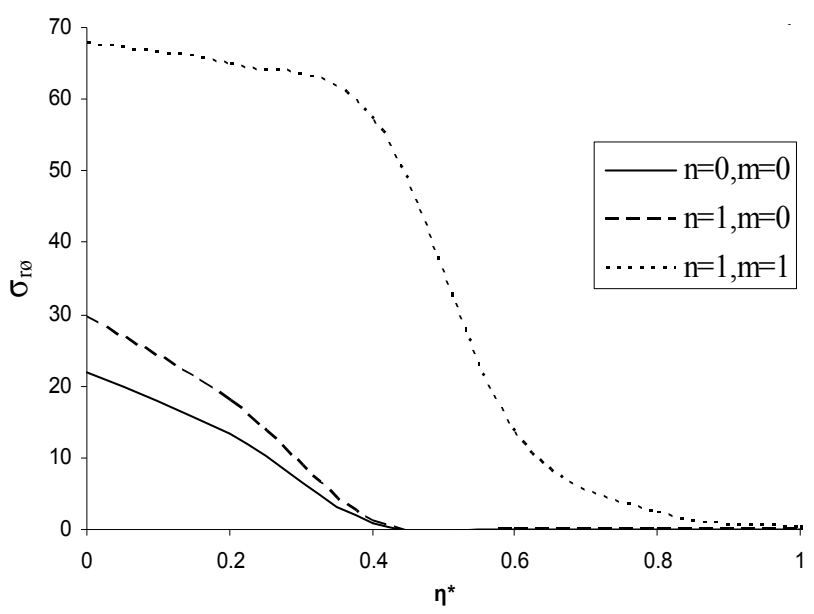

Figure 7. Variation of stress $\left(\sigma_{r \phi}\right)$ verses $\left(\eta^{*}\right)$ of hollow sphere.

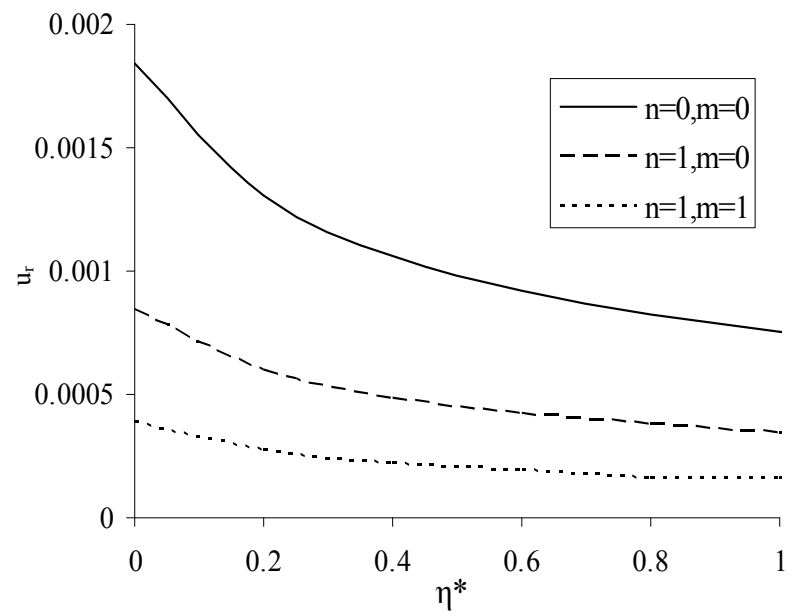

Figure 8. Variation of displacement $\left(u_{r}\right)$ verses $\left(\eta^{*}\right)$ of hollow sphere.

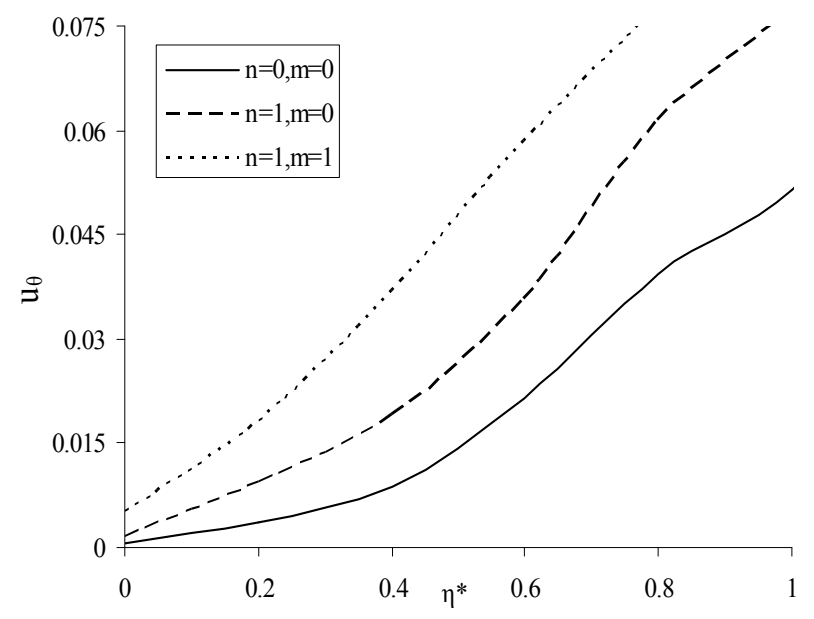

Figure 9. Variation of displacement $\left(u_{\theta}\right)$ verses $\left(\eta^{*}\right)$ of hollow sphere.

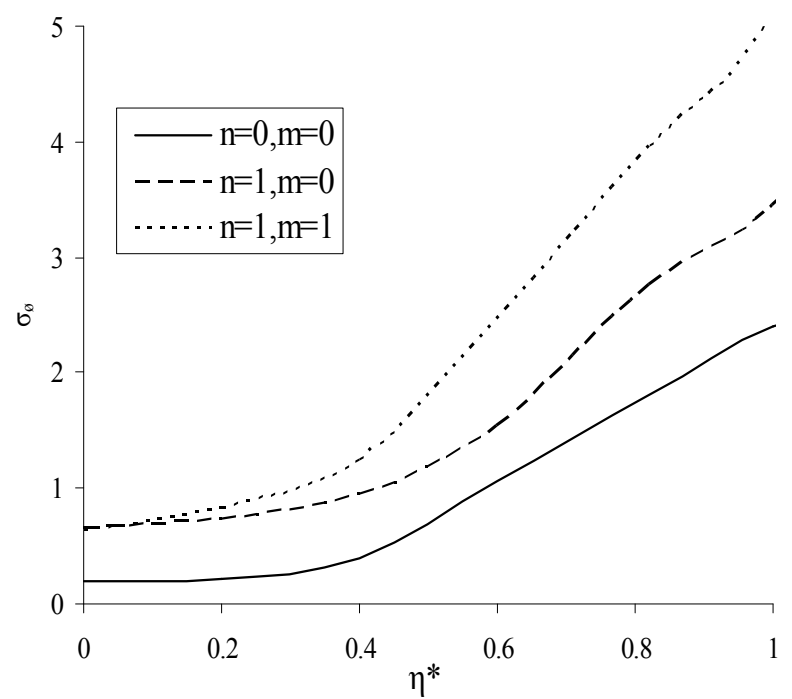

Figure 10. Variation between displacement $\left(u_{\phi}\right)$ verses $\left(\eta^{*}\right)$ of hollow sphere.

variations of these quantities show increasing trends with respect to increasing values of $\eta^{*}$ for all the modes of vibrations.

\subsection{Numerical Data/Information}

Here in Table 1 shows the Comparison of lowest frequency $(\widehat{\Omega})$ and Dissipation factor (D) in different media i.e. viscothermoelastic (VTE), thermoelastic (TE), viscoelastic (VE) and elastic (E) materials of hollow sphere and Table 2 has been given to check the validity of the numerical data by using t-test at fixed degree of harmonics $(n=1,2,3)$ for different values of thickness to mean radius ratio i.e. $\left(t^{*}=0.2,0.4,0.6,0.8,1.0\right)$ of hollow sphere.

\subsection{Statistical Analysis}

In this sub section we have performed some statistical analysis of computed data for lowest frequency and dissipation factor of three harmonics $(n=1,2,3)$ of spheroidal vibrations in hollow sphere of different thickness to mean radius ratio $(t *)$ made from VTE, TE, VE and $\mathrm{E}$ materials. The t-test has been used during the analysis in order to examine the influence of thermal, viscous and both thermal and viscous, effects on the vibrations.

\subsubsection{Lowest Frequency}

We take two samples $X$ and $Y$, where $X$ denotes lowest frequency $(\hat{\Omega})$ of each mode of vibrations in viscothermoelastic (VTE), thermoelastic (TE) or viscoelastic (VE) hollow spheres and $Y$ represents lowest frequency $(\hat{\Omega})$ of elastic (E) hollow sphere. The size of the sample in each case is $n_{1}=5$. Let $\mu_{V T E}, \mu_{T E}, \mu_{V E}$ and $\mu_{E}$ denote 
Table 1. Comparison of lowest frequency $(\hat{\mathbf{\Omega}})$ and dissipation factor (D) of vibrations of a hollow sphere with thickness to mean radius ratio at $t^{*}=0.6$.

\begin{tabular}{|c|c|c|c|c|c|}
\hline \multirow{2}{*}{ Quantity } & \multirow{2}{*}{ Harmonics (n) } & \multicolumn{4}{|c|}{ Hollow sphere } \\
\hline & & VTE & TE & VE & E \\
\hline \multirow{6}{*}{$\begin{array}{l}\text { Lowest } \\
\text { frequency } \\
(\hat{\Omega})\end{array}$} & 1 & 3.12531 & 4.13562 & 3.78694 & 2.78934 \\
\hline & 2 & 5.03378 & 5.40891 & 5.72728 & 5.86775 \\
\hline & 3 & 7.15597 & 5.32162 & 11.8138 & 6.23351 \\
\hline & 4 & 13.9483 & 16.97901 & 20.2419 & 13.097 \\
\hline & 5 & 28.0503 & 33.74073 & 27.3885 & 22.8264 \\
\hline & 6 & 47.3415 & 51.2391 & 34.1007 & 26.8179 \\
\hline \multirow[t]{10}{*}{ Quantity } & 7 & 55.1117 & 61.0285 & 39.3571 & 29.5775 \\
\hline & 8 & 65.60864 & 70.8765 & 43.93481 & 36.5856 \\
\hline & 9 & 74.49489 & 87.28608 & 48.6033 & 42.6692 \\
\hline & 10 & 80.9732 & 97.6551 & 58.6359 & 48.1659 \\
\hline & \multirow{2}{*}{ Harmonics (n) } & \multicolumn{4}{|c|}{ Hollow sphere } \\
\hline & & VTE & $\mathrm{TE}$ & $\mathrm{VE}$ & \\
\hline & 1 & 0.620948 & 0.99483 & 0.45198 & \\
\hline & 2 & 1.34792 & 1.79812 & 1.13429 & \\
\hline & 3 & 2.51173 & 2.34876 & 1.13425 & \\
\hline & 4 & 2.10415 & 2.4755 & 2.09345 & \\
\hline \multirow{6}{*}{$\begin{array}{l}\text { Dissipation } \\
\text { factor } \\
\text { (D) }\end{array}$} & 5 & 2.65234 & 3.24513 & 2.21375 & \\
\hline & 6 & 3.25564 & 3.90647 & 2.6234 & \\
\hline & 7 & 3.65919 & 5.06335 & 2.86333 & \\
\hline & 8 & 4.08234 & 6.82812 & 3.24512 & \\
\hline & 9 & 4.63452 & 7.36345 & 3.51789 & \\
\hline & 10 & 5.620948 & 7.65767 & 4.08993 & \\
\hline
\end{tabular}

mean values of the data representing to (VTE), (TE), (VE) and (E) hollow spheres, so that $\bar{X}=\mu_{V I E}, \mu_{T E}$ or $\mu_{V E}$ and $\bar{Y}=\mu_{E}$. The standard deviation $s\left(\mu_{V I E}, \mu_{I E}\right.$ and $\left.\mu_{V E}\right)$ is calculated by using formula

$s=\sqrt{\frac{1}{n_{1}+n_{1}-2}\left[\sum(X-\bar{X})^{2}+(Y-\bar{Y})^{2}\right]}$ in each case for all the considered modes. In order to test the null hypothesis $H_{0}=\mu_{V T E}=\mu_{T E}=\mu_{V E}=\mu_{E}$ for the consistency of means of the data pertaining to each case, we use t-test in which the t-statistic is computed from the relation

$$
t=\frac{\bar{X}-\bar{Y}}{s \sqrt{\left(\frac{2}{n_{1}}\right)}}
$$

The computed values if $t$ are given below $n=1: t_{1}=0.56, t_{2}=1.35, t_{3}=0.75$ $n=2: t_{1}=0.49, t_{2}=0.67, t_{3}=0.28$
Table 2. Numerical data of lowest frequency and dissipation factor of first three harmonics $(n=1,2,3)$ for different values of thickness to mean radius ratio $\left(t^{*}\right)$.

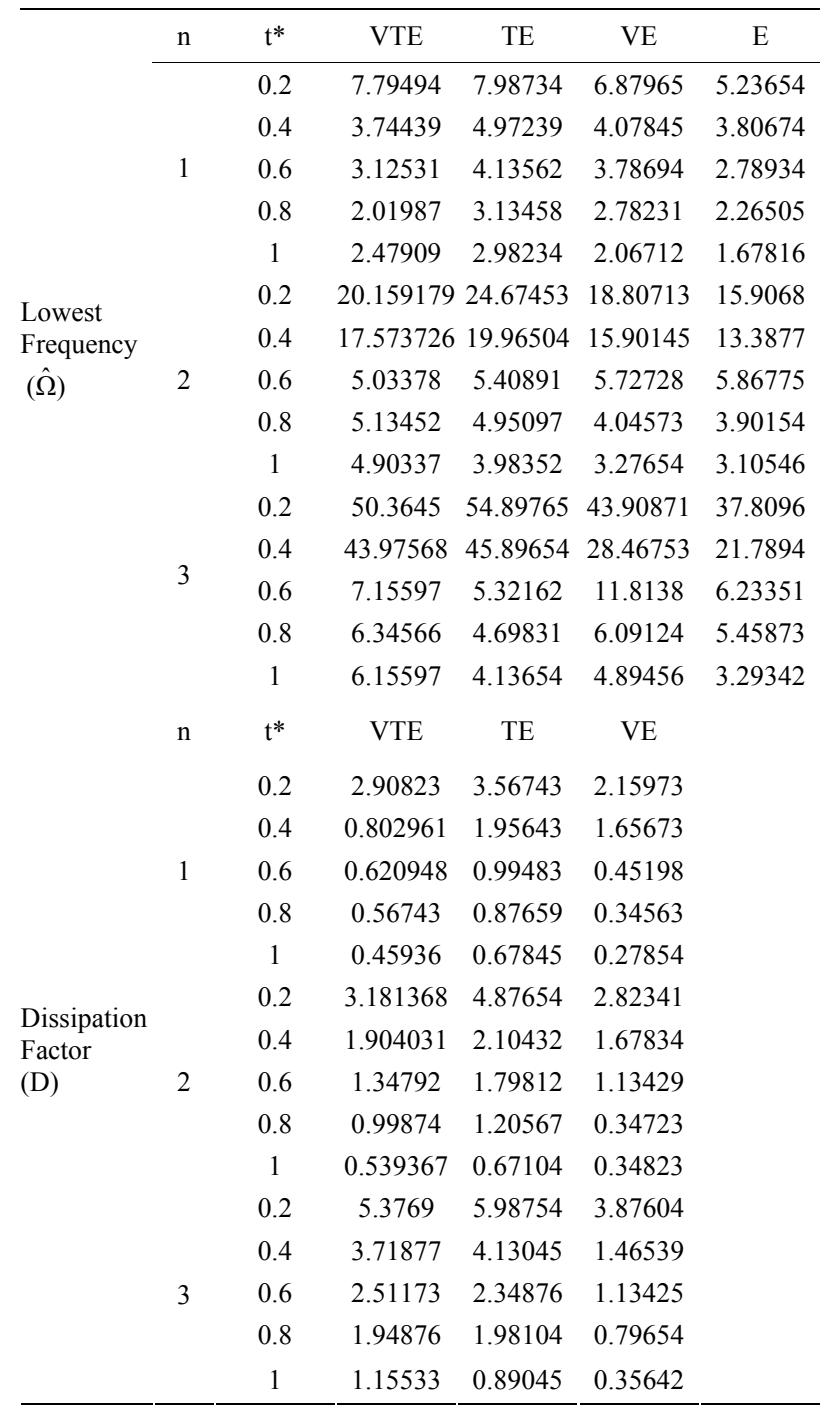

$n=3: t_{1}=0.77, t_{2}=0.62, t_{3}=0.41$

The tabulated values of $t$ for 8 degree of freedom at $1 \%$ level of significance is given by $t_{0.01}=3.36$, clearly $t_{i}<t_{0.01},(i=1,2,3)$ in each case and hence the null hypothesis $H_{0}$ is accepted at $1 \%$ level of significance. Thus the numerical data is consistent.

\subsubsection{Dissipation Factor}

Here $X$ denotes the dissipation factor (D) of (VTE) or (TE) hollow spheres and $Y$ represents the dissipation factor (D) of (VE) hollow spheres. The size of the sample is again $n_{1}=5$ and the null hypothesis $H_{0}=\mu_{V T E}=\mu_{T E}=\mu_{V E}$ is tested for (VTE) and (TE) spheres versus (VE) one by adopting above procedure. The computed values of the $\mathrm{t}$-statistic are given below: 
$n=1: t_{1}=0.15, t_{2}=0.96$

$n=2: t_{1}=0.51, t_{2}=1.51$

$n=3: t_{1}=1.49, t_{2}=1.42$

Since $t_{i}<t_{0.01},(i=1,2)$ in each case and hence the null hypothesis $H_{0}$ for is accepted at $1 \%$ level of significance. Thus the numerical data is consistent.

\section{Conclusion}

After simplifying the system of equations of motion and heat conduction of linear coupled viscothermoelasticity for a homogenous isotropic hollow sphere with the help of Helmholtz decomposition theorem, the extended power series (Matrix Fröbenius) method is successfully used to solve the resulting system of equations. It is noticed that toroidal vibrations (T-modes) get decoupled from rest of the motion and remain free of temperature variations as expected. The lowest frequency of spherical vibrations is noticed to be significantly affected due to temperature variations in a hollow sphere of copper material. This study may be useful in tribology and geophysical industrial applications.

\section{REFERENCES}

[1] W. Q. Chen, J. B. Cai, G. R. Ye and H. J. Ding, "On Eigen Frequencies of an Anisotropic Sphere," Journal of Applied Mechanics, Vol. 67, No. 2, 2000, pp. 422-424. doi: $10.1115 / 1.1303803$

[2] A. E. H. Love, "A Treatise on the Mathematical Theory of Elasticity," Dover Publications, New York, 1944.

[3] H. Cohen, A. H. Shah and C. V Ramakrishan, "Free Vibrations of a Spherically Isotropic Hollow Sphere," Acustica, Vol. 26, 1972, pp. 329-333.

[4] H. J. Ding, C. W. Qiu and L. Z. Hong, "Solutions to Equations of Vibrations of Spherical and Cylindrical Shells," Applied Mathematics and Mechanics, Vol. 16, No. 1, 1995, pp. 1-15. doi:10.1007/BF02453770

[5] H. W. Lord and Y. Shulman, "A Generalization of Dynamical Theory of Thermoelasticity," Journal of the Mechanics and Physics of Solids, Vol. 15, No. 5, 1967, pp.
299-309. doi:10.1016/0022-5096(67)90024-5

[6] E. Green and K. A. Lindsay, "Thermoelasticity," Journal of Elasticity, Vol. 77, No. 1, 1972, pp. 1-7. doi:10.1007/BF00045689

[7] R. B. Hetnarski and J. Ignaczac, "Generalized Thermoelasticity: Closed Form-Solutions," Journal of Thermal Stresses, Vol. 16, No. 4, 1993, pp. 473-498. doi:10.1080/01495739308946241

[8] G. R. Buchanan and G. R. Ramirez, "A Note on the Vibration of Transversely Isotropic Solid Spheres," Journal of Sound and Vibration, Vol. 253, No. 3, 2002, pp. 724732. doi:10.1006/jsvi.2001.4054

[9] J. N Sharma and N. Sharma, "Three-Dimensional Free Vibration Analysis of a Homogeneous Transradially Isotropic Thermoelastic Sphere," Journal of Applied Mechanics, Vol. 77, No. 2, 2010, pp. 1-9. doi:10.1115/1.3172141

[10] J. L. Neuringer, "The Fröbenius Method for Complex Roots of the Indicial Equation," International Journal of Mathematical Education in Science and Technology, Vol. 9, No. 1, 1978, pp. 71-72. doi: $10.1080 / 0020739780090110$

[11] C. Hunter, "Visco-Elastic Waves in Progress in Solid Mechanics," Wiley Inter-Science, New York, 1960.

[12] W. Flugge, "Visco-Elasticity," Blasdell, London, 1960.

[13] S. Mukhopadhyay, "Effect of Thermal Relaxation on Thermoviscoelastic Interactions in Unbounded Body with Spherical Cavity Subjected Periodic Load on the Boundary," Journal of Thermal Stresses, Vol. 23, No. 7, 2000, pp. 675-684. doi:10.1080/01495730050130057

[14] J. N. Sharma, "Some Considerations on the Rayleigh-Lamb Wave Propagation in Visco-Thermoelastic Plates," Journal of Vibration and Control, Vol. 11, No. 10, 2005, pp. 1311-1335. doi:10.1177/1077546305058267

[15] R. S. Dhaliwal and A. Singh, "Dynamic Coupled Thermoelasticity," Hindustan Publishing Corporation, New Delhi, 1980.

[16] C. G. Cullen, "Matrices and Linear Transformation," Addison-Wesley Publishing Company, Reading, 1966.

[17] H. Ding, W. Chen and L. Zhang, "Elasticity of Transversely Isotropic Materials,” Springer, Amsterdam, 2006. 


\section{Appendix}

The non-zero elements $L_{i j},(i, j=1,2,3)$ of the matrix $D_{1}^{*}$ in Equation (34) are given by

$$
\begin{aligned}
L_{13} & =\frac{H_{22}\left(p_{j}+1\right) H_{13}^{\prime}\left(p_{j}\right)-H_{12}\left(p_{j}+1\right) H_{23}^{\prime}\left(p_{j}\right)}{H_{11}\left(p_{j}+1\right) H_{22}\left(p_{j}+1\right)-H_{12}\left(p_{j}+1\right) H_{21}\left(p_{j}+1\right)} \\
L_{23} & =\frac{H_{21}\left(p_{j}+1\right) H_{13}^{\prime}\left(p_{j}\right)-H_{11}\left(p_{j}+1\right) H_{23}^{\prime}\left(p_{j}\right)}{H_{11}\left(p_{j}+1\right) H_{22}\left(p_{j}+1\right)-H_{12}\left(p_{j}+1\right) H_{21}\left(p_{j}+1\right)} \\
L_{31} & =\frac{H_{31}^{\prime}\left(p_{j}\right)}{H_{33}\left(p_{j}+1\right)} \\
L_{32} & =\frac{H_{32}^{\prime}\left(p_{j}\right)}{H_{33}\left(p_{j}+1\right)}
\end{aligned}
$$

The non-zero elements $K_{i j},(i, j=1,2,3)$ and $K_{i j}^{\prime},(i, j=1,2,3)$ of the matrix $D_{2 k+2}^{*}$ and $D_{2 k+1}^{*}$ defined in Equation (37) are obtained as

$$
\begin{gathered}
K_{11}=\frac{H_{31}^{\prime}\left(p_{j}+2 k\right) H_{13}^{\prime}\left(p_{j}+2 k+1\right)}{H_{33}\left(p_{j}+2 k+1\right)}+\frac{i \Omega^{-1}}{\delta_{0}^{*}} \frac{H_{22}\left(p_{j}+2 k\right)}{H^{*}\left(p_{j}+2 k\right)} \\
K_{12}=\frac{H_{32}^{\prime}\left(p_{j}+2 k\right) H_{13}^{\prime}\left(p_{j}+2 k+1\right)}{H_{33}\left(p_{j}+2 k+1\right)}-\frac{i \Omega^{-1}}{\delta_{0}^{*}} \frac{H_{12}\left(p_{j}+2 k\right)}{H^{*}\left(p_{j}+2 k\right)}
\end{gathered}
$$

$$
K_{21}=\frac{H_{31}^{\prime}\left(p_{j}+2 k\right) H_{23}^{\prime}\left(p_{j}+2 k+1\right)}{H_{33}\left(p_{j}+2 k+1\right)}+\frac{i \Omega^{-1}}{\delta_{0}^{*}} \frac{H_{21}\left(p_{j}+2 k\right)}{H^{*}\left(p_{j}+2 k\right)}
$$

$$
K_{22}=\frac{H_{32}^{\prime}\left(p_{j}+2 k\right) H_{23}^{\prime}\left(p_{j}+2 k+1\right)}{H_{33}\left(p_{j}+2 k+1\right)}+\frac{i \Omega^{-1}}{\delta_{0}^{*}} \frac{H_{11}\left(p_{j}+2 k\right)}{H^{*}\left(p_{j}+2 k\right)}
$$

$$
\begin{aligned}
K_{33}= & \frac{H_{31}^{\prime}\left(p_{j}+2 k+1\right) K_{1}^{*}}{H^{*}\left(p_{j}+2 k+1\right)}+\frac{H_{32}^{\prime}\left(p_{j}+2 k+1\right) K_{2}^{*}}{H^{*}\left(p_{j}+2 k+1\right)} \\
& +\frac{\tau_{0}^{*^{\prime}}}{H_{33}\left(p_{j}+2 k\right)}
\end{aligned}
$$

$$
\begin{aligned}
K_{1}^{*}= & \left(H_{22}\left(p_{j}+2 k+1\right) H_{13}^{\prime}\left(p_{j}+2 k\right)\right. \\
& \left.-H_{12}\left(p_{j}+2 k+1\right) H_{23}^{\prime}\left(p_{j}+2 k\right)\right)
\end{aligned}
$$

$$
\begin{aligned}
K_{2}^{*}= & \left(H_{21}\left(p_{j}+2 k+1\right) H_{13}^{\prime}\left(p_{j}+2 k\right)\right. \\
& \left.+H_{11}\left(p_{j}+2 k+1\right) H_{23}^{\prime}\left(p_{j}+2 k\right)\right)
\end{aligned}
$$

$$
\begin{aligned}
H^{*}\left(p_{j}+2 k\right)= & H_{11}\left(p_{j}+2 k\right) H_{22}\left(p_{j}+2 k\right) \\
& -H_{21}\left(p_{j}+2 k\right) H_{12}\left(p_{j}+2 k\right)
\end{aligned}
$$

$$
K_{32}^{\prime}=\frac{\left(-H_{31}^{\prime}\left(p_{j}+2 k\right) H_{21}\left(p_{j}+2 k\right)+H_{31}^{\prime}\left(p_{j}+2 k\right) H_{11}\left(p_{j}+2 k\right)\right)}{H^{*}\left(p_{j}+2 k\right)}+\frac{H_{32}^{\prime}\left(p_{j}+2 k+1\right) \tau_{0}^{*^{\prime}}}{H_{33}\left(p_{j}+2 k+1\right)}
$$

$$
\begin{aligned}
& D_{2 k+1}^{*} \\
& =-\left(H_{1}\left(p_{j}+2 k+1\right)\right)^{-1} \times\left[H_{2}\left(p_{j}+2 k\right) D_{2 k}^{*}+H D_{2 k-1}^{*}\right], \\
& k=0,1,2,3, \cdots
\end{aligned}
$$

where

$$
Z_{k}=\left[\begin{array}{c}
A_{k} \\
B_{k} \\
D_{k}
\end{array}\right]=\left\{\begin{array}{l}
D_{2 k+2}^{*} Z_{0} ; k \text { for even numbers } \\
D_{2 k+1}^{*} Z_{0} ; k \text { for odd numbers }
\end{array}\right.
$$

$D_{2 k+1}^{*}$

$=-\left(H_{1}\left(p_{j}+2 k+1\right)\right)^{-1}$

$\times\left[H_{2}\left(p_{j}+2 k\right) D_{2 k}^{*}+H D_{2 k-1}^{*}\right], k=0,1,2,3, \cdots$ 